\title{
Doped photopolymers for volume holographic applications
}

\author{
Shiuan Huei Lin, June-Hua Lin ${ }^{1}$, Yi-Nan $\mathrm{Hsiao}^{2}$ and Ken Y. Hsu ${ }^{1}$ \\ Department of Electrophysics, \\ ${ }^{1}$ Department of Photonics \& Institute of Electro-Optical Engineering, \\ ${ }^{2}$ Department of Materials Science and Engineering, \\ National Chiao Tung University \\ 1001 Ta Hsueh Road, Hsinchu, Taiwan 300, R.O.C. \\ Tel: 03-571-2121x56173; E-mail: lin@cc.nctu.edu.tw
}

\begin{abstract}
We fabricate two phenanthrenequinone-doped copolymers which can improve the holographic recording characteristics of phenanthrenequinone-doped poly(methyl-methacrylate) (PQ/PMMA) photopolymer. In these materials, the polymer matrix of $\mathrm{PQ} / \mathrm{PMMA}$ is modified to be copolymers, which composed of either poly(methyl-methacrylate-co -trimethylolpropane-triacrylate) or poly(methyl-methacrylate-co-acrylic acid 2-phenoxyethyl ester), respectively. With the chemical analyses of these materials before and after light exposure, we investigate the physical mechanism of the holographic recording in those copolymer samples. In addition, the holographic characteristics of different samples, including dynamic range and sensitivity, have been measured. These experimental results demonstrate that modification of the monomers is an efficient method to improve the material properties.
\end{abstract}

Keywords: Photopolymer, Copolymer, Volume hologram, Holographic materials.

\section{INTRODUCTION}

Holographic dada storage has been considered as a promising information storage technology because of its distinct page oriented data format for parallel data recording/retrieval. [1] It is known that to achieve high storage density, thousands of data pages are multiplexed in one spatial location of a storage volume by using Bragg selectivity of thick hologram. [2] Thus, a recording material of several-millimeter thick and with high photoinduced refractive index change is necessary. Recently, a few start-up companies, such as InPhase and Aprilis, have commercialized photopolymer materials for write-once holographic recording due to their success in developing materials with low shrinkage effect during holographic exposure. [3-4] It is crucial because otherwise the material shrinkage would induce dimensional distortion on the recorded grating and lead to a mismatch of Bragg condition for volume holograms such that the recorded information can not be retrieved completely [5]. To minimize the shrinkage effect, we have developed a technique for fabricating phenanthrenequinone-doped poly(methyl methacrylate) (PQ/PMMA) photopolymer of centimeter thick and with satisfactory optical quality [6-8]. Holographic experiments using these PQ:PMMA samples showed that the shrinkage coefficient of material is less than $10^{-5}$, such that our samples are especially attractive for the holographic data storage. [6]

Our strategy for alleviating the shrinkage problem is to use a two-step pre-polymerization technique to fabricate the material. In this technique, most of the MMA molecules are thermally polymerized using thermo-initiator, 2,2-Azo-bis-isobutyrolnitrile (AIBN), to form a PMMA polymer matrix and only a few percentages of un-reacted MMA monomer are left. The polymerization of monomers is used to form a strong polymer matrix for supporting material structure and provide high optical quality of the medium. On the other hand, the photosensitive element, PQ molecules are also added in liquid MMA monomer during material preparation. The photosensitizers together with residual monomers are dispersed uniformly in the polymer matrix, and they provide a mechanism to produce photochemical reaction when illuminated by the light exposure during holographic recording. Thus, the material is able to record phase holograms, whereas the basic photopolymer structure will not be affected by holographic recording such that the material shrinkage can be minimized.

One of drawbacks in our PQ:PMMA photopolymer is low recording sensitivity and small dynamic range of recording, because the concentrations of elements involved with photo-recording are limited. To further improve the material, investigations on the physical mechanism of holographic recording have been made. [9-11] The detail

Photorefractive Fiber and Crystal Devices: Materials, Optical Properties, and Applications XII, edited by Francis T. S. Yu, Ruyan Guo, Shizhuo S. Yin, Proc. of SPIE Vol. 6314, 631407, (2006)

0277-786X/06/\$15 - doi: 10.1117/12.678982

Proc. of SPIE Vol. $6314631407-1$ 
chemical analyses of PQ:PMMA material show that under light illumination, photon excites the quinone double bond on the carbonyl functional group of PQ molecule to make it becomes radical. The radical then reacts with the carbonic double bond on the vinyl group on the MMA molecule to form a new compound, which is less conjugated than the original molecular structure such that the refractive index of the material changes, and the refractive index pattern follows light intensity pattern during holographic recording. This message could be used to enhance combination ability of monomer molecules with PQ radicals. It may be achieved by increasing the number of vinyl functional groups on the monomer and/or changing the side functional group of the vinyl group on the monomer. In this paper, we chose two monomers, Trimethylolpropane Triacrylate (TMPTA) and Acrylic Acid 2-Phenoxyethyl Ester (PEA) to be added with MMA. The former (TPMPTA) has three vinyl groups and the later (PEA) has an additional benzene side functional group. Figure 1 shows their chemical formula. Our experiments show that these two monomers could not form a hard polymer matrix without use of MMA monomer. Thus, we have fabricated two kinds of co-polymer samples with 8:2 weight-ratio of MMA and the new monomer, one at a time. In the following, we first describe the fabrication of these two photopolymer materials. The holographic recording characteristics, such as dynamic range and sensitivity, of different samples are then presented. The results show that an improvement of PQ:PMMA can be achieved as additional monomer has been added.

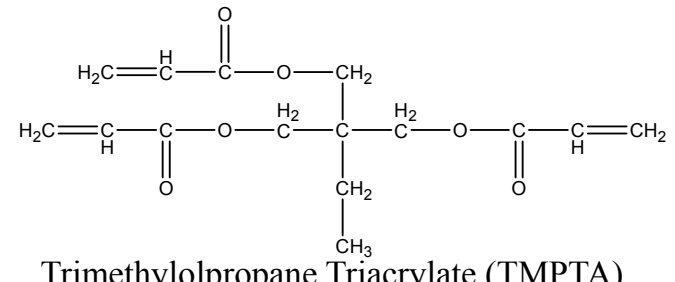

Trimethylolpropane Triacrylate (TMPTA)<smiles>C=CC(=O)OCCOc1ccccc1</smiles>

Acrylic Acid 2-Phenoxyethyl Ester(PEA)

Figure 1. The molecular structures of TMPTA and PEA

\subsection{Sample fabrications}

\section{MATERIAL FABRICATION AND ANALYSES}

To make 2-mm thick sample, 19.7 wt. \% of liquid PEA or TMPTA monomer were mixed with 78.8 wt. \% of liquid MMA monomer, respectively. Then, up to $0.7 \mathrm{wt} \%$ of photo-sensitizer, PQ molecule and $1 \mathrm{wt}$. \% of thermal initiator, 2,2-Azo-bis-isobutyrolnitrile (AIBN) molecule were dissolved in mixture solutions. The resulting solutions were placed in an ultrasonic water bath at $30{ }^{\circ} \mathrm{C}$ for $1 \mathrm{hr}$ until all components were dissolved completely. The solution was continuously stirred at $30^{\circ} \mathrm{C}$ for an appropriate period of time until it reached a suitable state of relative viscosity. Table I lists the stirred time of different mixtures. For comparison, the solution with $0.7 \mathrm{wt} \%$ of PQ and pure MMA monomer was also prepared by the same procedures. It should be noted that the PQ concentration listed in the Table 1 represents the saturated concentration of PQ molecule in each solution. As illustrated in Table I, the solution mixture with additional monomer TMPTA needs a shortest stirring period to reach designated viscosity. It indicates that with more vinyl groups, the sample has faster polymerization rate. On other hands, we anticipate that the addition of TMPTA can help to increase the glass temperature of final polymer matrix. After stirring, the viscous solutions were poured into the glass container with a 2 -mm thick spacer. The samples were then baked at $45{ }^{\circ} \mathrm{C}$ for $72 \mathrm{hr}$ until most of monomers were polymerized. They were removed from containers and formed bulk samples. Hereafter, the samples of PQ doped in polymer system of PMMA, copolymer system of Poly(TMPTA and MMA), and copolymer system of Poly(PEA and MMA) are

\begin{tabular}{|c|c|c|}
\hline Name & $\begin{array}{c}\text { PQ } \\
\text { concentration } \\
(\mathrm{wt} \%)\end{array}$ & $\begin{array}{c}\text { Stirring time } \\
\text { (Hr.) }\end{array}$ \\
\hline PQ/PMMA & 0.7 & 24 \\
\hline $\begin{array}{c}\text { PQ/PTMPTA } \\
\text {-co-PMMA }\end{array}$ & 0.5 & 6 \\
\hline $\begin{array}{c}\text { PQ/PPEA-co- } \\
\text { PMMA }\end{array}$ & 0.7 & 48 \\
\hline
\end{tabular}

Table 1 Stirring time of each mixed solution named as PQ/PMMA, PQ/PTMPTA-co-PMMA, and PQ/PPEA-co-PMMA photopolymers, respectively. 


\subsection{Chemical Analyses}

2.2.1 Thermal gravimetric analysis (TGA): We first confirm the existence of residual monomers in all of three photopolymers, which is a key requirement for holographic recording with these materials. This is done by performing thermal gravimetric analysis (TGA) of material. Since the molecular sizes of monomer and polymer matrix are dramatically different, their thermal de-composition temperatures are different. Thus, the ratio of the residual monomer in photopolymers can be determined by observing the dynamics of weight loss as temperature of material is increased. We designed a two-step heating procedure in use of DuPont Thermal Gravimetric Analyzer (model: TGA-Q500). In the first step, the temperature of material was increased with a rate of $2^{\circ} \mathrm{C} / \mathrm{min}$ until it reached the glass transition point of the sample, and the temperature was held for $800 \mathrm{~min}$. The glass transition point of each sample was measured to be at $110{ }^{\circ} \mathrm{C}$ for PQ/PPEA-co-PMMA photopolymer and at $127{ }^{\circ} \mathrm{C}$ for PQ/PMMA and PQ/PTMPTA-co-PMMA photopolymers, respectively. At these temperatures, the polymer matrix has not reached to thermal decomposition point, but the bonding becomes loose such that the monomer molecules can be evaporated from the bulk sample to cause a weight loss of material. Thus, the ratio of the weight loss at this stage is related to the ratio of the residual monomer in each photopolymer sample. In the second step, the temperature was increased to $800{ }^{\circ} \mathrm{C}$ at a rate of $10^{\circ} \mathrm{C} / \mathrm{min}$. Since the thermal decomposition temperature of our polymer material is lower than $800{ }^{\circ} \mathrm{C}$, we anticipate a two-stage weight loss dynamic. This behavior is induced by degradation of small molecules and/or polymer matrix phenomenon during material heating. The experimental results for three different samples are shown in Fig. 2(a). It is seen that all three samples show a thermally induced weight change in two stages. In the first stage that occurs at temperatures below thermal decomposition temperature of polymer matrix, the weight decreases with a lower rate and the weight loss is about $8 \%$. In the second stage that occurs at higher temperatures, the weight of material decreases with larger rate for the rest of $92 \%$ weight. We can consider the weight decrease in low temperature regime be attributed to the thermally induced loss of monomer, PQ and oligomers, which all are small molecules. The weight decrease in high temperature regime can be attributed to the loss of polymer. It implies that in three samples about $92 \%$ of monomer molecules are polymerized to form polymer matrix (during pre-thermo-polymerization) and less than $8 \%$ of molecules remain to be the residual monomer.

To know the details of weight loss in the first heating step ratio, we can enlarge the vertical axis in Fig. 2(a), as shown in Fig. 2(b). For comparison, the TGA of pure PQ molecule has been included and is shown by the symbol $\nabla$. It can be seen that for three photopolymers only $\sim 2 \%$ of weight of the material has been lost in the initial stage. This weight loss is not induced by sublimation of $\mathrm{PQ}$ molecules because it will not occur until at $\sim 155^{\circ} \mathrm{C}$, as indicated by the drastic weight loss of pure PQ shown in the figure. On the other hand, for all samples, the weight remains a constant until it decreases when the temperature rises to above $155^{\circ} \mathrm{C}$. We anticipate that occurrence of this second weight loss is attributed to the sublimation of PQ and oligomers. Thus, we summarize that the weight ratio of residual monomer in all of our photopolymer samples is about $\sim 2 \%$ and the PQ molecules together with oligomers contribute the rest weight loss of $6 \%$ in the first stage of weight loss process during thermal gravimetric analysis. These $\sim 2 \%$ in weight of residual monomers together with the PQ molecules could be responsible for holographic recording.
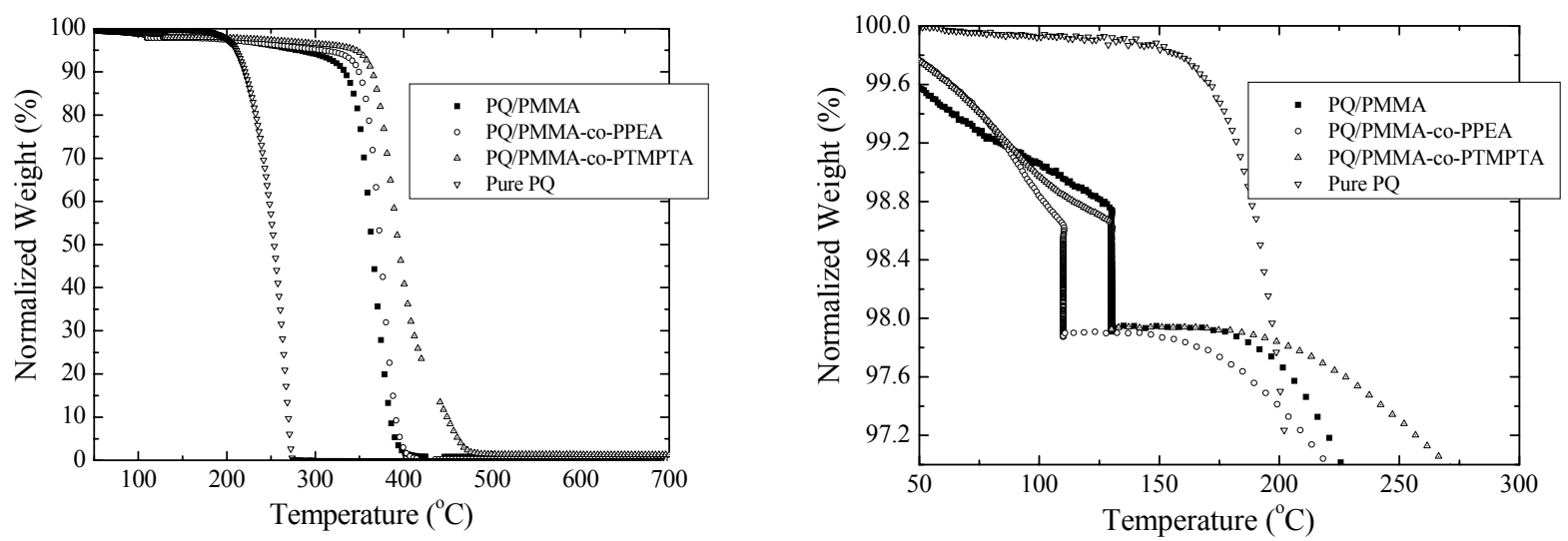

Fig. 2. The experimental results of thermal gravimetric analysis in three samples 
2.2.2 Mass spectra measurement (Mass): Next, it is necessary to know the species of residual monomers in three samples and the photoproduct after exposure. This information provides important information to understand the physical mechanism of holographic recording. An intuitive method is to investigate composition of material before and after light illumination. For this purpose, measurement of the mass spectral of different samples before and after light exposure is a useful method, because these spectra provides information about the changes of molecular weight distribution of the compounds in material, which can reveal occurrence of new compound produced by light exposure. In experiments, since our photopolymers were sensitive to green light, light exposure was produced by illuminating the samples with a 514-nm argon laser beam until the samples became transparent. Figure 3 shows the mass spectra of three samples. As illustrated in Fig. 3(a), a new characteristic peak signal occurs at 308 of molecular weight for the PQ/PMMA samples after exposure, which does not appear for the unexposed sample. Since the molecular weights of PQ and MMA are 208 and 100, respectively, the existence of peak signal at 308 gives evidence that the photoproduct is one MMA molecule attached to one PQ molecule in our PQ/MMA samples. This result is the same as our previous work shown in Ref. [10].

Figure 3(b) shows the measurement result for PQ/ PTMPTA-co-PMMA sample. It can be seen that a new peak at 308 of molecular weight also appears in the exposed sample. This indicates that the photo-chemical reaction in PQ/PTMPTA-co-PMMA sample is same as that in PQ/PMMA sample. Further, we can not find any peak that appears at around 296, which is the molecular weight of TMPTA molecule. This observation reveals that only one species of residual monomer, MMA, appears in PQ/PTMPTA-co-PMMA sample. From Fig. 3(c), it can be found that two new groups of peaks appear at neighboring ranges of 308 and 400 in the exposed PQ/PPEA-co-PMMA sample. Since the molecular weights of PQ and PEA are 208 and 192, respectively, the result indicates photo-induced attachments in the exposed PQ/PPEA-co-PMMA sample include one PQ molecule-to-one residual MMA and one PQ molecule-to-one
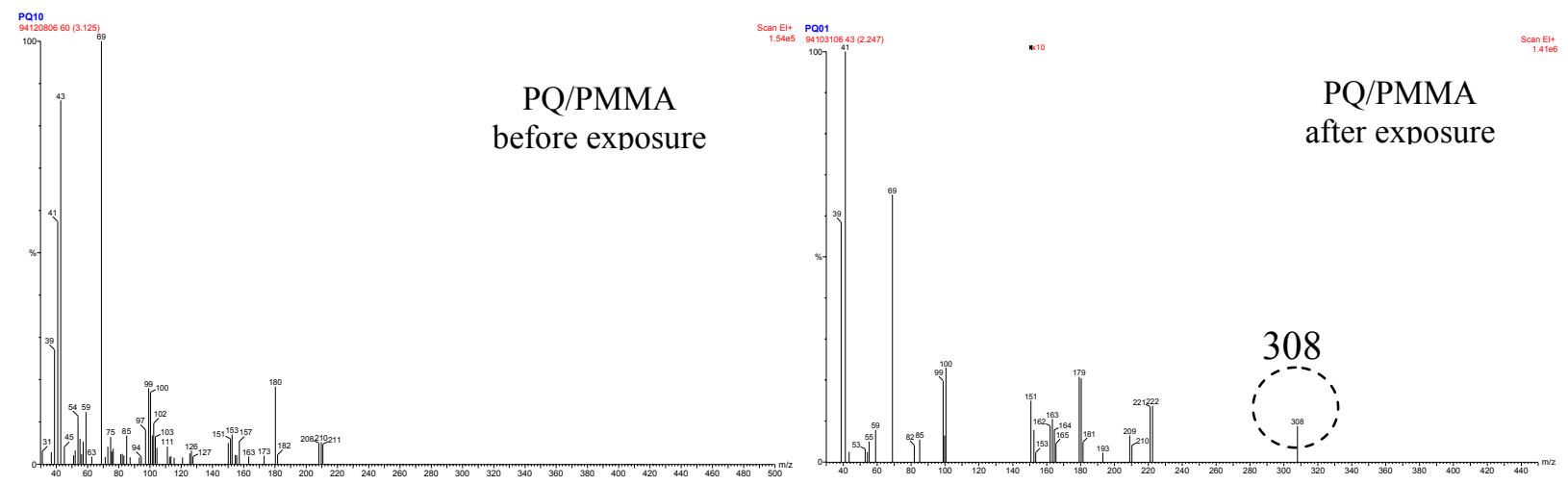

Fig. 3(a) The mass spectra of PQ/PMMA samples before and after exposure
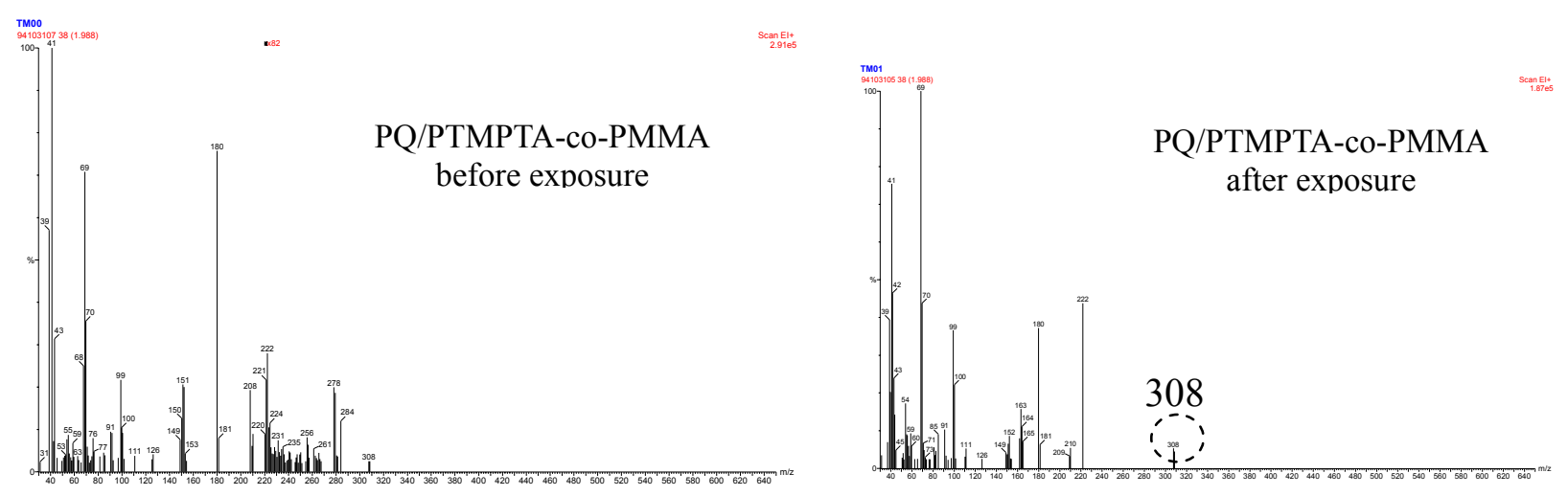

Fig. 3(b) The mass spectra of PQ/PTMPTA-co-PMMA samples before and after exposure 

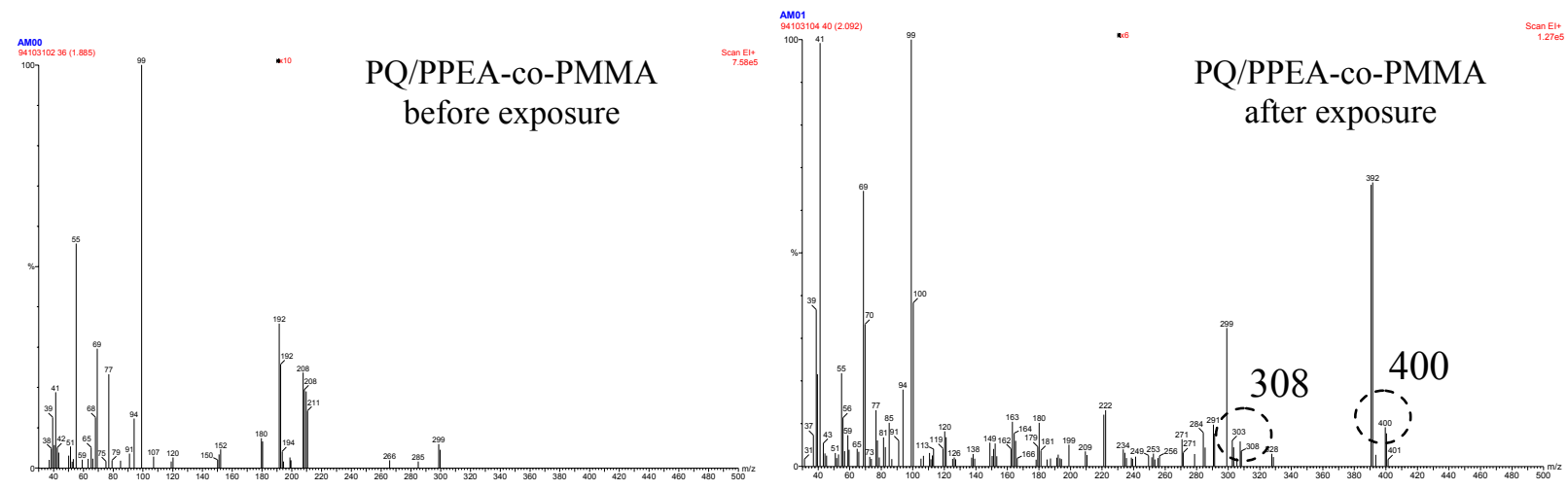

Fig. 3(c) The mass spectra of PQ/PPEA-co-PMMA samples before and after exposure

PEA monomer. Furthermore, in the spectrum of before-exposed sample there are two peaks appearing at 100 and 192 of molecular weight. This indicates that two kinds of residual monomers, MMA and PEA exist in the PQ/PPEA-co-PMMA sample.

In summary, figure 3 shows an important characteristic of light exposure for all three samples, i.e. a new compound with a molecular weight equals to one PQ plus one residual monomer has been formed. It indicates that the photoproduct is produced by attachment of one PQ molecule to one residual monomer molecule. Hence, we can confirm that the physical mechanism of holographic recording in three samples does not involve photochemical reaction of polymer matrix. Thus the photo-chemically induced shrinkage effect can be minimized. This is most important characteristic of these materials.

\section{EXPERIMENTAL CHARACTERIZATIONS}

\subsection{Holographic recording in three samples}

To demonstrate the ability of hologram recording in our photopolymer samples, we have recorded a plane-wave hologram in each sample by using S-polarized beams derived from an argon laser with wavelength of $514 \mathrm{~nm}$. The laser with intensity of $60 \mathrm{~mW} / \mathrm{cm}^{2}$ was collimated and split into two beams. Two beams were incident into the samples symmetrically with an intersection angle of $30^{\circ}$ outside the sample. After a series of experiments of different recording angle, we found that the samples have wide spatial frequency response. The recording angle is not critical to affect the holographic performance of material. During the recording a hologram, the diffraction efficiency, which is defined as the ratio of the intensity of the diffracted beam to intensity of summation of diffracted and transmitted beams, was measured in real-time by use of a weak 632.8-nm He-Ne-laser beam at the Bragg-matched angle. The experimental results are shown in Fig. 4. Diffraction efficiency for the different samples is plotted as a function of exposure energy. The diffraction efficiency of hologram recorded in PQ /PMMA sample is indicated by curve with symbol $\mathbf{m}$. It is seen that the diffraction efficiency first reaches a maximum at the exposure energy of $2.5 \mathrm{~J} / \mathrm{cm}^{2}$ and then it begins to

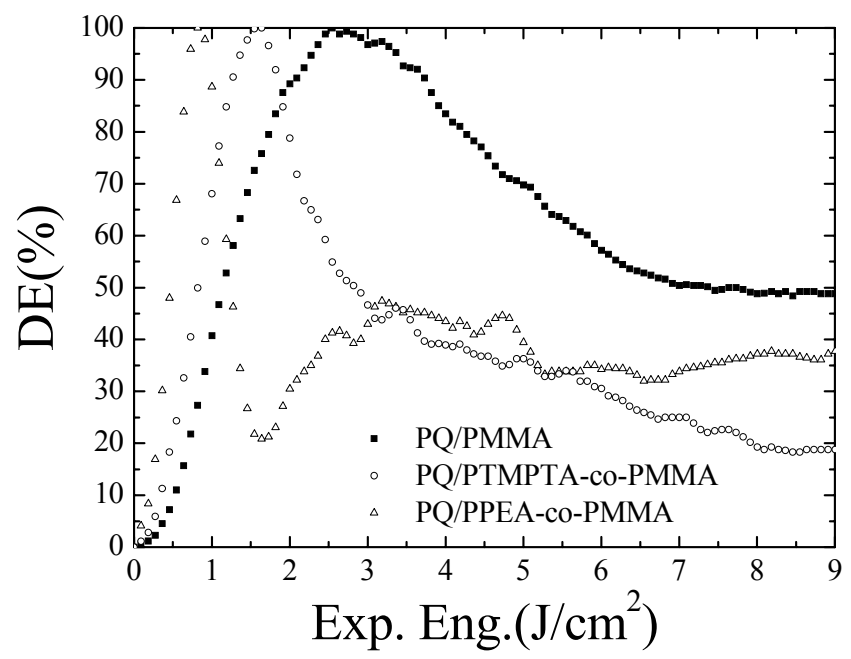

Fig. 4. The dynamics of holographic recording in three 
When the diffraction signal started to decrease from the maximum, it was observed that the transmitted beams become blurred and distorted. This phenomenon arises because of the formation of fanning noise gratings. The noise gratings come from the scattering of the recording light into the dark region of interference pattern during holographic recording.

Copolymer samples, PQ/PTMPTA-co-PMMA and PQ/PPEA-co-PMMA exhibit similar temporal behaviors of diffraction efficiency, as indicated in Fig. 4 by the symbols $\circ$ and $\Delta$, respectively. It is seen that the rising slope of diffraction efficiency in the early regime of holographic recording $\left(<2 \mathrm{~J} / \mathrm{cm}^{2}\right)$ is different for three samples. PQ/PPEA-co-PMMA sample has the largest one, which indicates that this sample has the fastest holographic recording speed. On the other hand, the rising slope for PQ/PMMA is the smallest. Thus, addition of the second species of monomer into PQ/PMMA photopolymer could be a useful method to improve holographic recording sensitivity.

\subsection{Characteristics of holographic data storage}

Two important criterions for evaluating a data storage technique are storage density and data recording rate. These two parameters are basically determined by the holographic recording performance of the material. Firstly, the storage density is determined by how many holograms can be multiplexed and recorded at one location of the material. Since each of these holograms shares the refractive index change of this spot, the larger values of total value of refractive index change means the higher storage density. The total refractive index changes of material and is usually defined as $\mathrm{M} / \#$ of recording material. [12] Secondly, data recording rate is determined by how fast one hologram data page can be recorded into medium. In physics, it can be defined as the sensitivity of material. High sensitive material enables holographic recording using low power lasers with short exposure time. In experiments, these two parameters can be obtained by performing a multiple- plane-wave-hologram recording at single spot in material. By using multiplexing technique, a series of holograms were recorded at one location until the material was exhausted. After each recording, the diffraction efficiency of each hologram was measured. From the couple wave theory of volume hologram, the refractive index change of a weak hologram is proportional to the square root of the diffraction efficiency produced by this hologram. Hence, the summation of the square roots of the diffraction efficiency of each hologram forms a running curve of the cumulative refractive index change of material as a function of cumulative exposure energy, viz $\mathrm{C}(\mathrm{E})=\sum_{i=1}^{\mathrm{n}} \sqrt{\eta_{i}}$, where $\mathrm{E}$ is the cumulative exposure energy and $\mathrm{n}$ is the total number of holograms that have been recorded. When the material got exhausted, no more holograms can be recorded and thus $\mathrm{C}$ tends to be saturated, and its saturation value is equal to the $\mathrm{M} / \#$. On the other hand, we define material sensitivity as change of the cumulative refractive index change divided by the corresponding exposure energy of that hologram, viz $\mathrm{S}=\Delta C(E) /\left.\Delta E\right|_{\text {one hologram }}$.

For all three samples, we have recorded 175 plane-wave holograms on a spot of the material with a peristrophic multiplexing system [13], in which each hologram was recorded with equal exposure energy $0.6 \mathrm{~J} / \mathrm{cm}^{2}$. The running curves for different samples are shown in Fig. 5. If we perform a curve fitting by the function $C(E)=C_{\text {sat }}\left[1-\left(E / E_{\tau}\right)\right]$, then the $\mathrm{M} \#$ (i.e. $\mathrm{M} \#=C_{\text {sat }}$ ) and a characteristic energy constant $E_{\tau}$ of the material are obtained. From these results, sensitivity can be evaluated. Without loss of generality, we consider the recording sensitivity of a fresh sample. During this stage, the cumulative exposure energy is small, thus the exponential function $\mathrm{C}(\mathrm{E})$ can be linearized and the material sensitivity $\mathrm{S}$ can be found to be $\mathrm{S}=\left.\frac{d C(E)}{d E}\right|_{E \rightarrow 0}=\frac{M \#}{E_{\tau}}$.

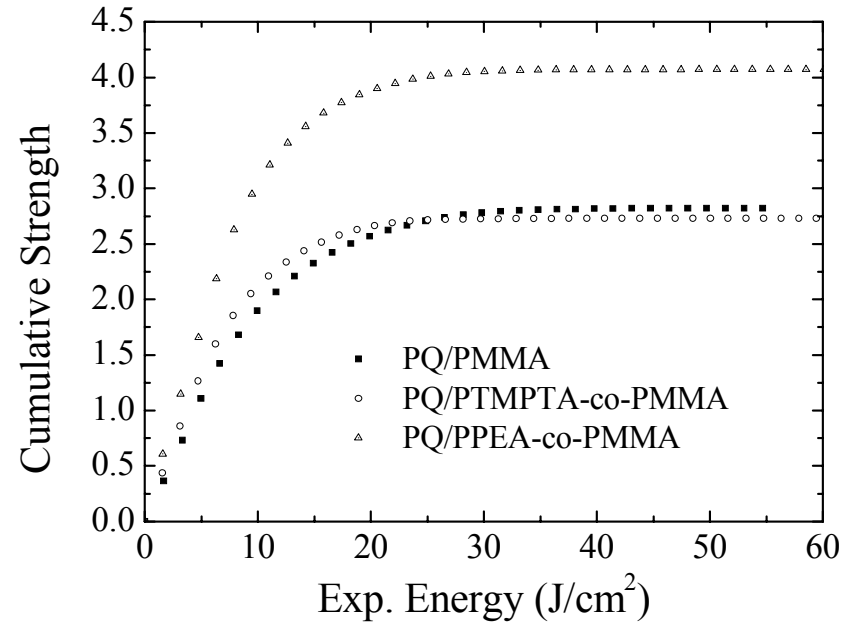

Fig. 5. The dynamics of multiple hologram recording in three different samples. 
By curve fitting plots in Fig.5, the material M\#, $E_{\tau}$ and $S$ of materials for the three samples are estimated and summarized in Table 2 . It can be seen in the table that the M/\# of PQ:PPEA-co-PMMA is 4.11, which is 1.4 times larger than that of PQ:PMMA (M\# 2.87). On the other hand, the $\mathrm{M} / \#$ of PQ:PTMPTA-co-PMMA is slightly smaller than that of PQ:PMMA. However, the characteristic energy constant, $E_{\tau}$ of PQ/PTMPTA-co-PMMA and PQ/PPEA-co-PMMA are 6.88 and $7.75 \mathrm{~J} / \mathrm{cm}^{2}$, respectively. Both are slightly smaller than that of PQ/PMMA $\left(\mathrm{E}_{\tau} \sim 9.3 \mathrm{~J} / \mathrm{cm} 2\right)$. As a result, the sensitivity of the former two samples has been improved, as shown in last column in Table 2. The results confirm

\begin{tabular}{|c|c|c|c|}
\hline Samples & $\begin{array}{c}\mathrm{E}_{\tau} \\
\left(\mathrm{J} / \mathrm{cm}^{2}\right)\end{array}$ & $\mathrm{M} \#$ & $\begin{array}{c}\text { Sensitivity } \\
\left(\mathrm{cm}^{2} / \mathrm{J}\right)\end{array}$ \\
\hline PQ/PMMA & 9.3 & 2.87 & 0.31 \\
\hline $\begin{array}{c}\text { PQ/PTMPTA } \\
\text {-co-PMMA }\end{array}$ & 6.88 & 2.76 & 0.4 \\
\hline $\begin{array}{c}\text { PQ/PPEA-co } \\
\text {-PMMA }\end{array}$ & 7.75 & 4.11 & 0.53 \\
\hline
\end{tabular}

Table 2. The $E_{\tau}, \mathrm{M} \#$ and sensitivity of three different samples.

our idea that by adding the second species of monomer to form copolymer structure, holographic recording characteristics of PQ:PMMA photopolymer can be improved. We can anticipate that the material might be further improved as appropriate monomer has been chosen.

\section{CONCLUSIONS}

We have fabricated three different PQ doped photopolymer samples and performed their chemical analyses. The TGA analyses indicate that they all have $\sim 2 \mathrm{wt} \%$ residual monomers after thermo-polymerization. The mass spectra measurements of the three samples before and after light exposure indicate that light exposure on the samples will induce a photochemical reaction between one PQ molecule and one residual monomer molecule. Thus, the physical mechanism of holographic recording in these samples follows our design strategy that the polymer matrix will not be affected in the light exposure process. As a result, photo-chemically-shrinkage effect in our samples can be anticipated to be minimized. In addition, the holographic recording characteristics of these samples have been measured. The results show that both the $\mathrm{M} / \#$ and the exposure sensitivity have been improved by introducing the second monomer into PQ/PMMA samples to form copolymer structure.

\section{ACKNOWLEDGEMENTS}

This research is supported in part from the Ministry of Education under Research Excellence Project Phase II (contract number: NSC95-2752-E-009-007-PAE) and MOE ATU Program, and in part from the National Science Council, Taiwan, under contract NSC95-2112-M-009-007. The authors are grateful for technical discussion with Prof. Hwa-Tsung Whang and for his help in providing samples and chemical analysis measurements.

\section{REFERENCES}

1. H. J. Coufal, D. Psaltis, G. Sincerbox, Holographic data storage (Springer series in optical sciences, 2000).

2. A. Pu and D. Psaltis, "High-density recording in photopolymer-based holographic three-dimensional disk," Applied Optics, 35, pp. 2389-2398, 1996.

3. For example, to see website of http://www.inphase-technologies.com/technology/whitepapers/index.html.

4. For example, to see website of http://www.aprilisinc.com/technology.htm.

5. M. L. Hsieh and K. Y. Hsu, "Grating detuning effect on holographic memory in photopolymers," Optical Engineering, 40, No. 10, pp. 2125-2133, 2001.

6. K. Y. Hsu, "Holographic data storage using photopolymer," in Proceeding of SPIE International Symposium on Photorefractive Fiber and Crystal Devices: materials, Optical Properties, and Applications V, SPIE Proc. 3801, pp.66-74, (Denver, Colorado, 18-23 July 1999). 
7. S. H. Lin, K. Y. Hsu, W. Z. Chen and W. T. Whang, "phenanthrenequinone-doped poly(methyl methacrylate) photopolymer bulk for volume holographic data storage," Optics Letters, 25, pp.451-453, 2000.

8. Ken Y. Hsu, Shiuan Huei Lin, and Yi-Nan Hsiao,“ Experimental characterization of phenanthrenequinone-doped poly(methyl methacrylate) photopolymer for volume holographic storage" Opt. Eng. 42, 1390, 2003.

9. J. Mumbru, I. Solomatine, D. Psaltis, S. H. Lin, K. Y. Hsu, W. Z. Chen, and W. T. Whang, "Comparison of the recording dynamics of phenanthrenequinone-doped poly(methyl methacrylate) materials," Optics Communications, 194, pp. 103-108, 2001.

10. Yi-Nan Hsiao, Wha-Tzong Whang, and Shiuan Huei Lin, "Analyses on physical mechanism of holographic recording in phenanthrenequinone-doped poly(methyl methacrylate) hybrid materials", Opt. Eng. 43(9), pp. 1993-2002, 2004.

11. S. Farid, D. Hess, G. Pfundt, K. H. Scholz, and G. Steffan, "Photoreactions of o-Quinones with Olefins: a New Type of Reaction leading to Dioxole Derivatives", Chemical Communication, 434, pp.638-639, 1968.

12. F. Mok, G. Burr, and D. Psaltis, "A system metric for holographic memory systems", Optics Letters, 21, pp.886-888, 1996.

13. K. Curtis, A. Pu and D. Psaltis, "A method for holographic storage using peristrophic multiplexing", Optics Letters, 19, pp.993-995, 1994. 